\title{
Bisphosphonates and primary breast cancer risk: an updated systematic review and meta-analysis involving 963,995 women
}

This article was published in the following Dove Press journal:

Clinical Epidemiology

\begin{abstract}
Yupeng Liu, I,2
Xiaosan Zhang, ${ }^{3}$

Hongru Sun, ${ }^{2}$ Shu Zhao, ${ }^{4}$

Yuxue Zhang, ${ }^{5}$ Dapeng Li, ${ }^{2}$

Qingyuan Zhang, ${ }^{4}$

Yashuang Zhao ${ }^{2}$

'Department of Preventive Medicine, School of Public Health and Management, Wenzhou Medical University, Wenzhou 325035,

People's Republic of China; ${ }^{2}$ Department of Epidemiology, Public Health School of Harbin Medical University, Harbin I5008I, People's Republic of China; ${ }^{3}$ Department of Internal Medicine, The Affiliated Cancer Hospital of Zhengzhou University, Henan Cancer Hospital, Zhengzhou 450008,

People's Republic of China; ${ }^{4}$ Department of Medical Oncology, The Third Affiliated Hospital of Harbin Medical University, Heilongjiang Cancer Hospital, Harbin I5008I, People's Republic of China; ${ }^{5}$ Department of Preventive Medicine, Public Health School of Harbin Medical University, Harbin I5008I, People's Republic of China
\end{abstract}

Correspondence: Yupeng Liu Department of Preventive Medicine, School of Public Health and Management, Wenzhou Medical University, II 2 Nanliu Road, Wenzhou, Zhejiang Province 325035, People's Republic of China Tel +8605778 6699182

Fax +86057786699182

Email liuyupenf@।26.com

Yashuang Zhao

Department of Epidemiology, Public Health School of Harbin Medical University, 157 Baojian Road, Harbin,

Heilongjiang Province 15008I, People's

Republic of China

Tel +86045I87502823

$\mathrm{Fax}+86045187502885$

Email zhao_yashuang@263.net
Importance: Prevention of primary breast cancer $(\mathrm{BCa})$ in women is of great public health importance. The existing results from observational epidemiologic studies focused on the association between bisphosphonates and primary $\mathrm{BCa}$ risk have been inconsistent.

Objective: To update this systematic review and meta-analysis to assess the effect of bisphosphonates on primary $\mathrm{BCa}$ risk.

Data sources: We comprehensively searched MEDLINE, EMBASE, Cochrane libraries, ProQuest, and Web of Science through June 25, 2018 for relevant studies.

Study selection: Epidemiological studies that assessed the effect of bisphosphonates on the risk of primary $\mathrm{BCa}$ in women.

Data extraction and synthesis: We reported this meta-analysis according to the PRISMA guidelines. Available multivariable-adjusted effect estimates and corresponding $95 \% \mathrm{CIs}$ were pooled with a random-effects model.

Main outcomes and measures: The prespecified main outcome was the risk of primary BCa. Results: In total, five cohort studies involving 657,558 women and 12,991 primary BCa patients, three population-based case-control studies involving 54,701 primary BCa cases and 237,962 healthy controls and two randomized controlled trials (RCTs) involving 13,774 women and 165 primary $\mathrm{BCa}$ patients were included in this meta-analysis. Bisphosphonates were associated with a $12 \%$ decreased risk of primary $\mathrm{BCa}(\mathrm{RR}, 0.88 ; 95 \% \mathrm{CI}, 0.83-0.94)$. However, when we analyzed study designs separately, the pooled results from observational studies were inconsistent with that from RCTs. The observed association of primary BCa risk with long-term use ( $\geq 1$ year) of bisphosphonates seemed to be more robust and stronger than that of short-term use $(<1$ year) (RR, 0.75; 95\% CI, 0.66-0.84; and 0.90; 95\% CI, 0.84-0.97; respectively).

Conclusion: This meta-analysis adds to the body of evidence for an association between bisphosphonates and a significantly decreased risk of primary BCa. However, future largescale RCTs are required to validate this concern.

Keywords: bisphosphonates, primary breast cancer risk, meta-analysis

\section{Introduction}

Breast cancer $(\mathrm{BCa})$ is the most frequently diagnosed cancer and the leading cause of cancer deaths among females worldwide, ${ }^{1}$ with an estimated 2,088,849 newly diagnosed cases in 2018. Incidence rates are highest in economically developed countries but are now increasing rapidly in developing countries as well. ${ }^{2}$ Prevention of primary $\mathrm{BCa}$ in women is of great public health importance. Bisphosphonates are widely used for the prevention and treatment of osteoporosis. ${ }^{3,4}$ Recently, an increasing body of evidence has shown that bisphosphonates have potential anticancer properties against 
the risk of developing primary $\mathrm{BCa}$. Although the precise biologic mechanisms that link bisphosphonates and $\mathrm{BCa}$ risk remain unclear, an increasing body of evidence supports the notion that bisphosphonates may modify the tumour cell microenvironment and directly affect tumour cells through a variety of pathways, including inhibition of osteolysis and release of bone-derived growth factors, ${ }^{5}$ inhibition of protein prenylation, ${ }^{6,7}$ and angiogenesis inhibition, 8,9 enhanced immune surveillance by activation of gamma delta T cells, ${ }^{10,11}$ direct inhibition of tumour cell growth, proliferation, adhesion and invasion, and induction of apoptosis. ${ }^{12,13}$

To date, several epidemiologic studies have assessed the relationship between bisphosphonates and $\mathrm{BCa}$ risk. However, the findings have been inconsistent. Recently, a report from two randomized controlled trials (RCTs, Fracture Intervention Trial (FIT) and Health Outcomes and Reduced Incidence with Zoledronic Acid Once Yearly-Pivotal Fracture Trial (HORIZON-PFT)) has showed that bisphosphonates could not decrease the incidence risk of primary $\mathrm{BCa}^{14}$ In our previous metaanalysis, which included four observational studies, we reported a significant protective effect of bisphosphonates on the risk of $\mathrm{BCa} .{ }^{15}$ Since then, however, three large cohort studies ${ }^{16-18}$ involving 95,701 participants and 1,079 $\mathrm{BCa}$ patients and one large nested case-control study $^{19}$ involving 49,933 BCa patients and 232,780 controls have been published. We therefore performed an updated comprehensive systematic review and metaanalysis to determine and quantify the potential antitumor effect of bisphosphonates on the risk of primary $\mathrm{BCa}$.

\section{Methods}

\section{Search strategy and selection criteria}

This meta-analysis was registered on PROSPERO (CRD42014014901) and was reported according to the PRISMA guidelines (the checklist was provided as Appendix A). ${ }^{20}$ We systematically searched the electronic databases of MEDLINE, EMBASE, ProQuest, and Web of Science through August 15, 2016 to identify relevant publications. We used a combination of Medical Subject Headings (MeSH) terms and corresponding free-text terms as follows: (diphosphonates[MeSH Terms] or diphosphonate or bisphosphonate or alendron* or etidron* or clodron* or zoledron* or risedron* or ibandron* or pamidron* or tiludron* or neridron* or olpadron*) and (breast neoplasms[MeSH Terms] or breast neoplasm or breast cancer or breast tumor or mammary carcinoma or mammary neoplasm). The reference sections and citation lists of the retrieved literature, including original research articles, reviews, editorials, and letters, were manually reviewed for potentially relevant articles. An updated search was performed (from August 16, 2016, through June 25, 2018) to identify new literatures.

We included studies that met the following inclusion criteria: 1) epidemiological studies (including case-control or cohort studies, or RCTs) that addressed the potential effect of bisphosphonates on primary $\mathrm{BCa}$ risk; 2) studies that reported effect estimates, which included the RRs, ORs and HR with 95\% CIs, or reported sufficient information to calculate these values; 3) when multiple articles were published from the same population, we included the most recent or complete publication or the one with the highest quality score; and 4) studies that addressed the association between bisphosphonates and contralateral $\mathrm{BCa}$ risk in women with primary $\mathrm{BCa}$ were excluded from the main analysis but included in a sensitivity analysis. There were no restrictions on language, sample size, or participant characteristics.

\section{Data extraction and quality assessment}

The eligibility determination, data extraction, and quality assessment for each study were performed independently by two authors (YP.L. and XS.Z.). Disagreements were resolved by discussion and consensus with a third author (HR.S.). Standard electronic forms specifically created for the present study were used to record the following information: authors, publication date, characteristics of participants (country, age and menopausal status), bisphosphonate exposure (type, dose, frequency, and duration), and studies (study design, sample size, and the controlled or adjusted potential confounding factors). Both the maximally and minimally adjusted effect sizes with 95\% CIs were recorded, if available. The extracted data from each study was carefully checked and verified before performing metaanalysis. When necessary, we contacted authors of studies for missing information.

A quality-scoring system, which was partially derived from that developed by Voskuil and colleagues and included 15 items ( 5 on selection bias, 8 on misclassification bias, and 2 on confounding bias) with a maximum score of 95, was adopted to assess the methodological quality of observational epidemiological studies. ${ }^{21}$ The quality score of each study was presented as a percentage of the maximum score, and studies with a score of $>70 \%$ were categorized as highquality studies (Appendix B). The Jadad score $^{22}$ and 
Cochrane risk of bias tool ${ }^{23}$ were used to assess the quality of RCTs.

\section{Statistical analysis}

In this meta-analysis, the maximally adjusted effect sizes and $95 \%$ CIs were pooled using random-effects models, rather than fixed-effects models, to analyze the results in a conservative manner. When an individual study only reported effect estimates for each type of bisphosphonate separately ${ }^{24}$ or separately reported risk estimates in ductal carcinoma in situ and invasive $\mathrm{BCa},{ }^{25}$ likely to account for a higher relative weight in the pooled analysis, we initially combined the subgroup datasets into a study-specific effect estimate with a fixed-effects model. ${ }^{26}$ To evaluate whether a duration-dependent threshold effect of bisphosphonates on primary $\mathrm{BCa}$ risk exists, we conducted a cumulative metaanalysis based on the medication duration across the included studies. We estimated the between-study heterogeneity using the $Q$ test and the $I^{2}$ Statistic. ${ }^{27,28}$ To investigate potential sources of the between-study heterogeneity, we performed subgroup and meta-regression analyses using random-effects models.

For sensitivity analyses, we removed the most relatively weighted study from each subgroup analysis to assess its influence on the summarized estimates and to explore potential sources of heterogeneity. We then conducted another sensitivity analysis using the fixed-effects model. In addition, to test whether the underlying confounders that were adjusted or controlled in the original observational studies could have influenced the effect of bisphosphonates on primary $\mathrm{BCa}$ risk, we conducted repetition analyses using the minimally adjusted data and subsequently analyzed the confounding RR, which was defined as the ratio of the pooled results of the maximally and minimally adjusted data. ${ }^{29}$ Potential impact of an unmeasured confounder on the observed results was calculated presenting $E$-values for point estimates and the upper limit of the CIs as recently suggested by VanderWeele and colleagues. ${ }^{30}$ We performed a sensitivity analysis to assess the association between bisphosphonates and the risk of developing contralateral $\mathrm{BCa}$ among primary $\mathrm{BCa}$ patients. We also performed a sensitivity analysis to include only the prospective studies (including cohort studies, RCTs and nested case-control studies), because the prospective nature of cohort studies and RCTs is invaluable for confirming the temporal sequence of bisphosphonate use and primary $\mathrm{BCa}$ onset and therefore helps to examine causal associations.
To determine the evidence strength of the association between bisphosphonates and the incidence risk of primary $\mathrm{BCa}$, an evidence synthesis modified from previous studies was developed. ${ }^{21}$ To assess potential publication bias, we used funnel plots for asymmetry and, formally, used the Begg's rank correlation and Egger's linear regression tests. ${ }^{31}$ Furthermore, we robustly adjusted for the summarized results by applying the Duval and Tweedie's trim and fill method. ${ }^{32}$ We did all analyses with Comprehensive Meta Analysis version 2.2.046 (Biostat, Englewood, NJ, USA). All tests were two-sided. Statistical significance was defined as $P$-values $<0.05$.

\section{Results}

\section{Characteristics and reporting quality of included studies}

The search strategy identified 10 eligible studies (Figure 1). The main characteristics of 10 studies included are provided in Tables S1-S3. Briefly, there were five cohort studies, ${ }^{16-18,24,25}$ three population-based case-control studies $^{19,33,34}$ and two RCTs. ${ }^{14}$ The two RCTs enrolled 13,774 participants, with a total of 165 primary $\mathrm{BCa}$ cases. The five cohort studies enrolled 657,558 participants, with mean follow-up periods ranging from 2.9 to 8.5 years and 12,991 cases, while the three case-control studies included 54,701 cases and 237,962 controls. Overall, these 10 studies enrolled 67,857 primary BCa cases and 188,685 bisphosphonate users. Of these, seven studies ${ }^{14,18,24,25,33,34}$ focused on $\mathrm{BCa}$ risk, and the other three studies ${ }^{16,17,19}$ assessed the association between bisphosphonates and the risk of developing various cancers, including $\mathrm{BCa}$. Among these studies, the majority of the participants were postmenopausal women, and the mean age ranged from 54.2 to 73.5 years. All but one study ${ }^{33}$ reported the type of bisphosphonate used, of which alendronate was the most common (range, 51.8-100\%).

\section{Bisphosphonates and primary $\mathrm{BCa}$ risk}

Overall, we noted a $12 \%$ risk reduction of primary $\mathrm{BCa}$ in women who used bisphosphonates compared with non-users (RR, 0.88; 95\% CI, 0.83-0.94; Table 1 and Figure 2). Considering the study design separately, we found similar associations in cohort studies and case-control studies (OR, 0.83; 95\% CI, 0.71-0.98; RR, 0.87; 95\% CI, 0.80-0.95; respectively); however, the association was not found in RCTs (RR, 1.13; 95\% CI, 0.81-1.57). When restricted to invasive $\mathrm{BCa}$ or postmenopausal women, the pooled RRs 


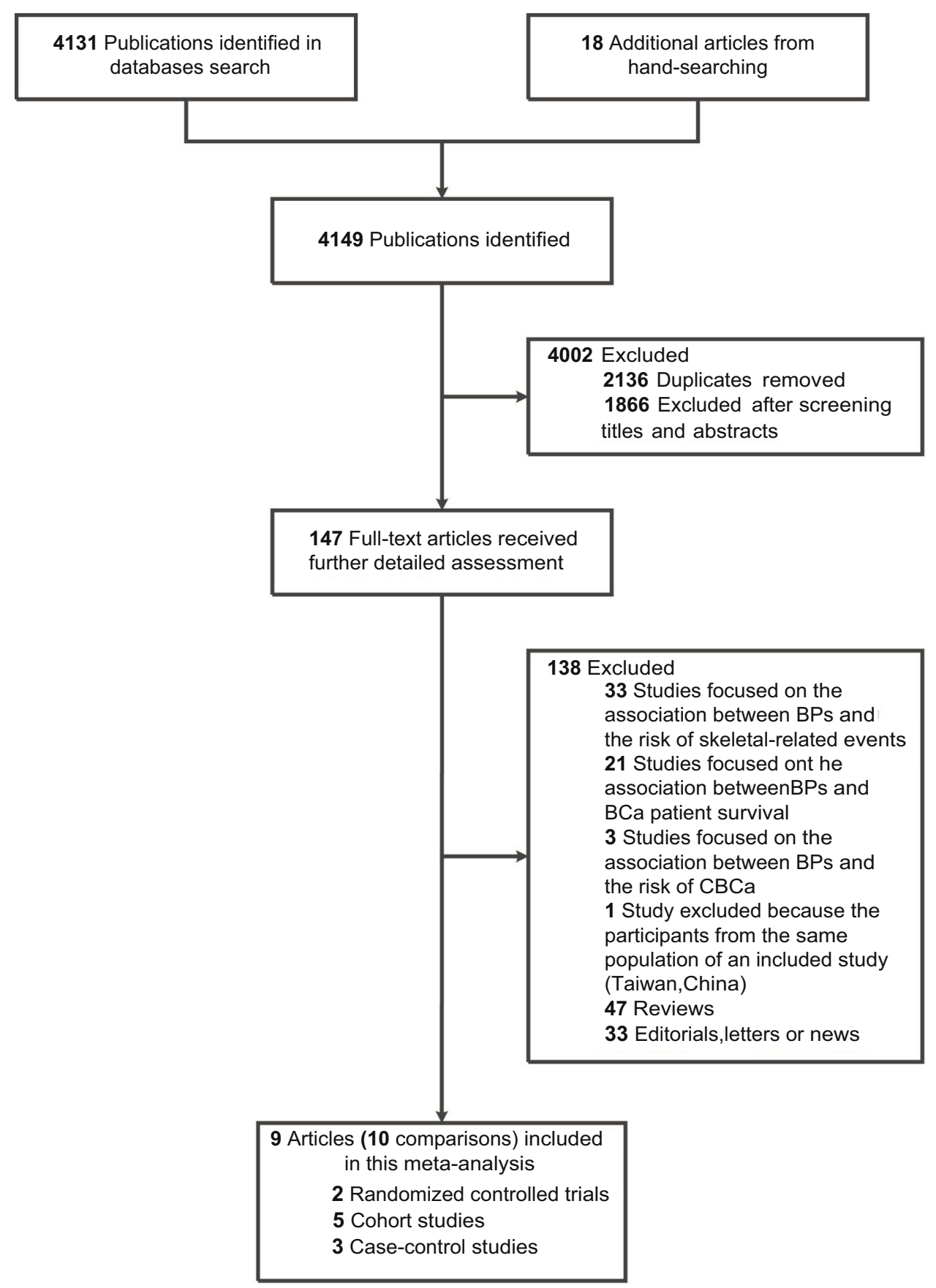

Figure I Flow diagram of study selection.

Abbreviations: $\mathrm{BCa}$, breast cancer; $\mathrm{BPs}$, bisphosphonates; $\mathrm{CBCa}$, contralateral breast cancer.

were 0.83 (95\% CI, 0.69-0.99; Figure S1) and 0.89 (95\% CI, 0.80-0.99; Figure S2), respectively.

\section{Duration-response analysis of} bisphosphonates and primary $\mathrm{BCa}$ risk

Eight studies ${ }^{14,17-19,25,33,34}$ were included in this durationresponse analysis. Subgroup analyses (Table 1 and Figure S3) showed that women who used bisphosphonates for $\geq 1$ year before diagnosis had a $25 \%$ reduced risk of $\mathrm{BCa}(\mathrm{RR}, 0.75$; 95\% CI, 0.66-0.84), whereas those who received bisphosphonates for $<1$ year had an attenuated risk reduction (RR, 0.90; $95 \%$ CI, 0.84-0.97). Similarly, cumulative meta-analysis based on duration of bisphosphonate use demonstrated that a $10 \%$ risk reduction of primary $\mathrm{BCa}$ was observed when the duration of medication usage reached 1 year (RR, $0.90 ; 95 \%$ CI, $0.84-0.97$; Figure S4). Thereafter, the observed protective effect did not 


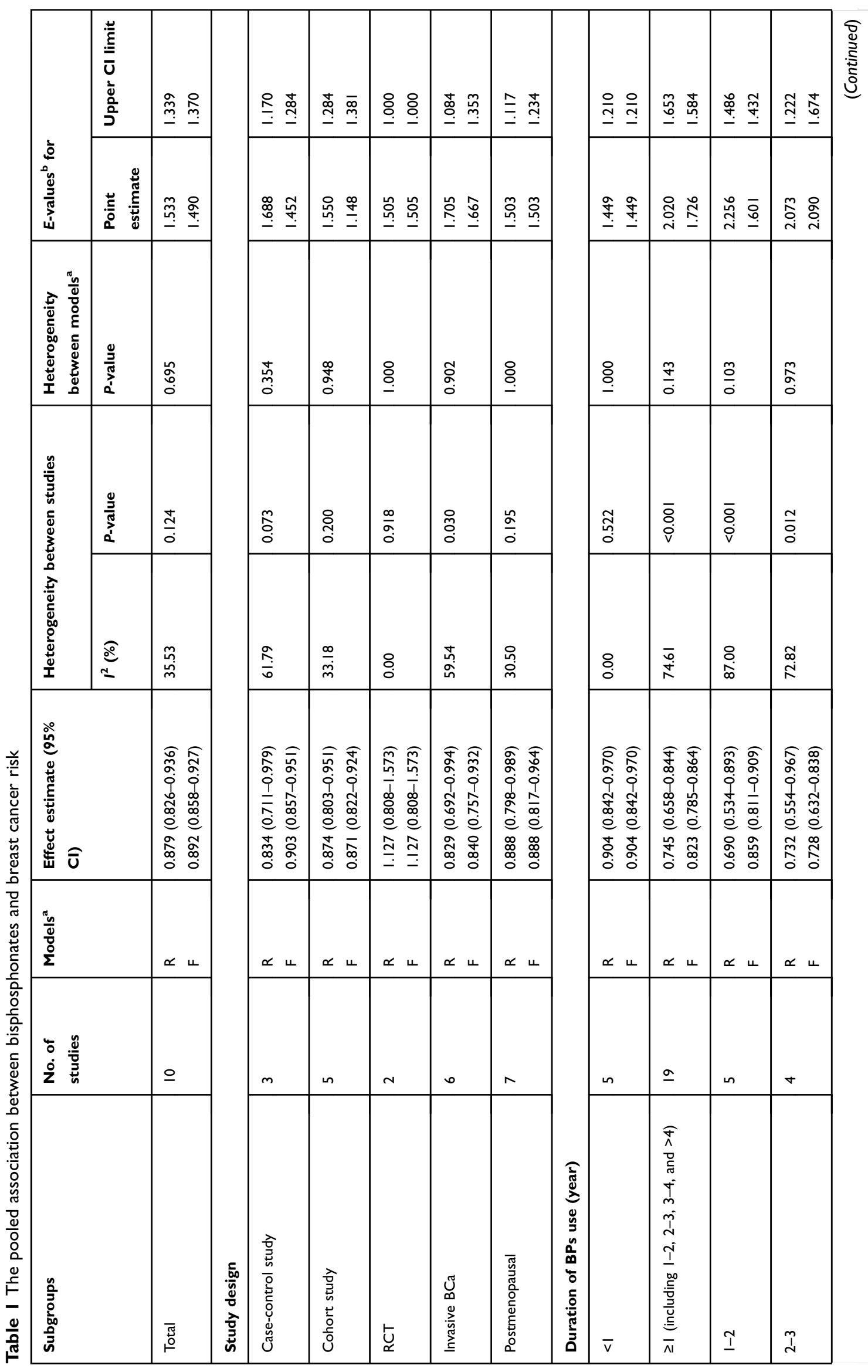




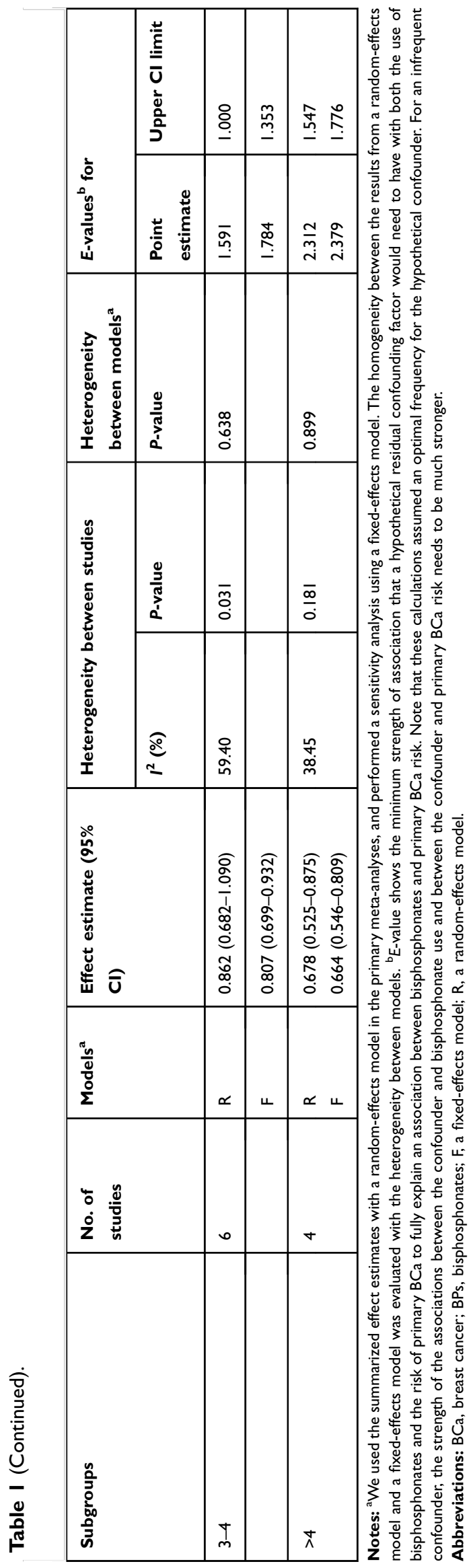

change substantially with increasing years of medication usage, although subsequent datasets have increased the precision of the magnitude of the effect.

\section{Sensitivity analyses}

We performed a repetition analysis with fixed-effects models and found no significant change in the pooled results (Table 1). Furthermore, a repetition analysis using the minimally adjusted data from original studies was also carried out, and confounding RRs demonstrated that the summarized results did not significantly differ from those with the maximally adjusted data (Table S4). Moreover, confounding RR values indicated that the summarized results with the maximally adjusted data were more conservative. According to the results from $E$-value sensitivity analyses, $E$-values for both the observed point estimates and the upper limit of the CIs were all small (Table 1), indicating that little unmeasured confounding would be needed to explain away the observed association estimates. Another sensitivity analysis excluding the study with the most relative weight in each subgroup did not result in a substantial change of the summarized results (Table S5), except for subgroup analyses of cohort studies, bisphosphonate use duration of $<1$ year, 2-3 years or $>4$ years. We also performed a sensitivity analysis by excluding retrospective case-control studies and found a RR of 0.90 (95\% CI, 0.85-0.95; Figure S5). Another sensitivity analysis that excluded the RCTs showed a RR of 0.87 (95\% CI, 0.82-0.93; Figure S6).

Another three observational studies assessed the impact of bisphosphonates on risk of second primary contralateral BCa among BCa survivors. ${ }^{35-37}$ The characteristics of these studies were shown in Tables S6 and S7. When these results were pooled together, the RR was 0.84 (95\% CI, 0.66-1.07; Figure S7). By including these studies, we performed another sensitivity analysis and found a RR of 0.88 (95\% CI, 0.84-0.93; Figure S8).

\section{Heterogeneity}

In general, there was no obvious between-study heterogeneity $\left(P=0.12 ; \quad I^{2}=35.53 \%\right)$. When considering study design, we observed moderate heterogeneity across the case-control studies $\left(P=0.07 ; I^{2}=61.79 \%\right)$, but not across the cohort studies $\left(P=0.20 ; I^{2}=33.18 \%\right)$ or the RCTs $\left(P=0.92 ; \quad I^{2}=0.00 \%\right)$. Based on results from metaregression analyses, we found that the between-study heterogeneity was not relevant to the mean age of participants ( $P=0.65$, Figure S9A), quality score $(P=0.76$, Figure $\mathrm{S} 9 \mathrm{~B})$, the number of $\mathrm{BCa}$ patients $(P=0.11$, Figure $\mathrm{S} 9 \mathrm{C})$, or bisphosphonate users ( $P=0.56$, Figure $\mathrm{S} 9 \mathrm{D})$. 


\begin{tabular}{|c|c|}
\hline Study & $\operatorname{RR}(95 \% \mathrm{Cl})$ \\
\hline Vinogradova et al, ${ }^{19} 2013$ & $0.92(0.87,0.97)$ \\
\hline Rennert et al, ${ }^{34} 2010$ & $0.80(0.65,0.99)$ \\
\hline Newcomb et al, ${ }^{33} 2010$ & $0.70(0.54,0.91)$ \\
\hline Subtotal_Case-control $\left(I^{2}=61.79 \%, P=0.07\right)$ & $0.83(0.71,0.98)$ \\
\hline Vestergaard et al, ${ }^{24} 2011$ & $0.86(0.80,0.93)$ \\
\hline Fournier et al,$^{18} 2017$ & $0.98(0.85,1.12)$ \\
\hline Cardwell et al, ${ }^{17} 2011$ & $0.75(0.63,0.89)$ \\
\hline Chlebowski et al, ${ }^{25} 2010$ & $0.90(0.72,1.12)$ \\
\hline Chiang et al, ${ }^{16} 2012$ & $0.92(0.66,1.28)$ \\
\hline Subtotal_Cohort $\left(I^{2}=33.18 \%, P=0.20\right)$ & $0.87(0.80,0.95)$ \\
\hline Hue et $\mathrm{al}^{1}{ }^{14} 2014-\mathrm{FIT}$ & $1.11(0.71,1.73)$ \\
\hline Hue et al, ${ }^{14} 2014$-HORIZON-PFT & $1.15(0.70,1.90)$ \\
\hline Subtotai_RCT $\left(I^{2}=0.00 \%, P=0.92\right)$ & $1.13(0.81,1.57)$ \\
\hline Overall $\left(I^{2}=35.53 \%, P=0.12\right)$ & $0.88(0.83,0.94)$ \\
\hline \multicolumn{2}{|l|}{ Test for overall effect: $z=-4.01 ; P<0.001$} \\
\hline Test for subgroup differences: $P=0.26$ & \\
\hline
\end{tabular}

Figure 2 Forest plot for the association between bisphosphonates and primary breast cancer risk. Square markers indicate effect sizes of each study; horizontal lines, the $95 \% \mathrm{Cl}$. The diamond data marker indicates the summarized effect size. The vertical solid line indicates the overall pooled effect. Note that the studies are ranked in order of their relative weights from random effects analysis.

\section{Publication bias}

We found limited evidence for publication bias using funnel plots (Figure S10), Begg's rank correlation or Egger's regression test (Table S8). In addition, the adjusted summary effect size analyzed using the trim and fill method did not demonstrate a substantial change, which also implies no evidence of publication bias.

\section{Discussion}

This meta-analysis has strengthened the evidence for anticancer effects of bisphosphonates in BCa primary prevention. We found a $12 \%$ risk reduction of $\mathrm{BCa}$ associated with bisphosphonates. However, according to the evidence synthesis, there is a general "indecisive" evidence for this association (Table S9).

Bisphosphonates have predominantly been used in women with osteoporosis who typically have a low bone mineral density (BMD) level, which is caused by a low level of circulating estrogen that is associated with $\mathrm{BCa}$ risk $^{38}$ and may result in confounding by indication in observational studies. ${ }^{14}$ Consequently, it is vital to determine whether the association of risk reduction of $\mathrm{BCa}$ with bisphosphonates is merely responsible for the inverse relationship between osteoporosis, the indication of bisphosphonate therapy, and BCa risk. Eight of the 10 analyzed studies have adjusted for BMD status ${ }^{14}$ or other factors that reflect the level of BMD between bisphosphonate users and non-users, including the 5-year hip fracture score $^{25}$ and history of osteoporosis or fracture. ${ }^{16-19,33}$ When we pooled the results of these eight studies together, we found that a decreased risk of $\mathrm{BCa}$ remained associated with the use of bisphosphonate, which supports the evidence for an authentic inverse association between bisphosphonates and $\mathrm{BCa}$ risk, independent of indications of bisphosphonates. Menopausal status can influence the levels of estrogens in women, as circulating concentrations of estrogens decrease markedly after menopause. ${ }^{39}$ Among the studies included, enrolled participants were predominantly postmenopausal women, and none of these studies reported an effect in premenopausal women separately. 
When we limited participants to postmenopausal women, we found a similar inverse association between bisphosphonates and $\mathrm{BCa}$ risk.

Two previous meta-analyses have focused on the same issues. In our previous meta-analysis that included only 4 studies, we reported a $15 \%$ risk reduction of primary $\mathrm{BCa}$ related with bisphosphonates. ${ }^{15}$ Thereafter, another metaanalysis by $\mathrm{Ou}$ et al reported a very similar effect estimate of $16 \%$ risk reduction associated with bisphosphonates. ${ }^{40}$ The pooled effect size of our current study is smaller than those in these two previous meta-analyses. The possible reason may be that neither of the previous meta-analyses had taken into account the RCT results.

As commonly known, results from observational epidemiological studies cannot confirm causality. ${ }^{41}$ However, it is possibly difficult to conduct an RCT to address the effect of bisphosphonates in the primary prevention of $\mathrm{BCa}$ due to ethical challenges and the requirement for a considerably large sample size. ${ }^{41}$ Recently, the results from two RCTs (FIT and HORIZON-PFT) were published. ${ }^{14}$ Hue and colleagues showed that 3-4 years of bisphosphonate treatment did not decrease the risk of invasive $\mathrm{BCa}$ in postmenopausal women. ${ }^{14}$ Of note, the two RCTs were at high risk of selective reporting bias according to the Cochrane Collaboration recommendation (Figures $\mathrm{S} 11 \mathrm{~A}$ and $\mathrm{B}$ ), since they were not initially designed to study $\mathrm{BCa}$ outcomes as the authors stated. ${ }^{14}$ The data about incident $\mathrm{BCa}$ were collected from adverse event databases, thus, the results were prone to detection bias. Because women assigned to bisphosphonate therapy group were more likely to visit clinics or be hospitalized and subsequently had higher incidence rate of newly diagnosed $\mathrm{BCa}$, since the number of patients with adverse events were significantly higher in the bisphosphonate treatment group compared with the placebo control group $(95.5 \%$ vs $93.9 \%, P=0.002){ }^{42}$ Additionally, given the limited number of BCa patients during the follow-up period and the limited statistical power (in the Supplementary), the findings from the two RCTs (even those that were very well done) are not sufficient to overturn the findings from observational studies.

Three observational studies assessed the impact of bisphosphonates on contralateral $\mathrm{BCa}$ risk among $\mathrm{BCa}$ survivors. $^{35-37}$ When pooling these results together, we did not find an obvious association, which is consistent with the results from a meta-analysis of individual patient data from RCTs that showed no significant effect on contralateral $\mathrm{BCa}^{43}$ These findings provide no support for bisphosphonate treatment as $\mathrm{BCa}$ chemoprevention strategy. It is supposed that bisphosphonates may have different effects on healthy women (as average-risk populations of developing $\mathrm{BCa}$ ) or on women with primary BCa.

Eight $^{14,17-19,25,33,34}$ of the studies included assessed the relationship between bisphosphonate use duration (from $<1$ to $>5$ years) and primary $\mathrm{BCa}$ risk, although the findings were inconsistent. The controversy has focused on the optimum duration of bisphosphonate use, the magnitude of the beneficial effect and possible harm from long-term use of bisphosphonates. Subgroup analyses based on the medication periods suggested that, compared to non-users, women who used bisphosphonates for $<1$ year had a $10 \%$ decreased risk of primary $\mathrm{BCa}$, whereas women with $\geq 1$ year of bisphosphonate use had a $25 \%$ lower risk of primary BCa. However, in the subgroup of $<1$ year of bisphosphonate use, a sensitivity analysis excluding Vinogradova' study, which had $84.37 \%$ relative weight, showed no significant effect of bisphosphonates on primary $\mathrm{BCa}$ risk. Given the limited number of studies included in each subgroup, the results from subgroup analyses should not be overemphasized. Consistent with the findings from subgroup analyses, cumulative meta-analyses showed that this observed beneficial effect became robust only when the treatment duration reached 1 year, and thereafter the effect tended to stabilize over time. Interestingly, this result is in accordance with both the anti-resorptive properties of bisphosphonates, which were only observed after at least 6 months of use, ${ }^{44}$ and the pharmacokinetics of bisphosphonates, by which, once entering bone, the drug sustains a stable anti-resorptive potency for several years. $^{45}$

Given the global use of bisphosphonates among millions of women over the past 20 years, it can be concluded that adverse effects related to bisphosphonates are relatively low. ${ }^{46}$ Commonly reported side-effects are mild and include dyspepsia, nausea, and oesophagitis, whereas serious sideeffects including osteonecrosis of the jaw, renal toxicity, and oesophageal cancer are rare. However, whether these serious side-effects are attributable to bisphosphonates alone remain inconclusive. ${ }^{26,47-49}$ Another concern is the longterm ( $>5$ years) side-effects of bisphosphonates, and few studies have addressed this issue. ${ }^{50}$ Additional welldesigned prospective studies are still needed to evaluate potential long-term adverse effects and determine whether the benefit of bisphosphonate therapy could outweigh possible disadvantages. Based on the current evidence, the benefits from bisphosphonate treatment for 1-5 years significantly outweigh the potential harm from these drugs. 
Unlike tamoxifen, which has previously been shown to be efficient in the primary and relapse prevention of $\mathrm{BCa}^{51-53}$ but has "exceptionally low" acceptance and prevalence of use for chemoprevention, ${ }^{51,54}$ bisphosphonates should have a substantial and extensive public health benefit for the prevention of primary $\mathrm{BCa}$ because bisphosphonates are already widely prescribed for the prevention and treatment of osteoporosis or cancer-induced bone $\operatorname{loss}^{10-13}$ and an increasing number of bisphosphonate users worldwide are expectable. For instance, approximately 40 million prescriptions for bisphosphonates are written every year in the United States alone. ${ }^{55}$

The current meta-analysis had some strengths. First, a large number of participants were enrolled in the studies included, which added validity to this analysis. Second, we performed extensive sensitivity analyses, including confounding RR, $E$-value and subgroup analyses, to test the robustness of our results. Finally, we performed subgroup analysis and metaregression analysis to explore the potential causes of heterogeneity. However, several limitations of this study should be considered. First, various types of bisphosphonates were given to women in the individual studies, which could have contributed to heterogeneity; of these drugs, alendronate, clodronate, and zoledronic acid were the major bisphosphonates prescribed. Although the type of bisphosphonate used may have differing antitumor activities between nitrogen-containing bisphosphonates and non-nitrogen-containing bisphosphonates, as demonstrated by mechanistic studies in vitro and in vivo models, ${ }^{56,57}$ we could not explore this differential effect due to the lack of available data. Although currently unclear, researches on which bisphosphonates are better at reducing the risk of primary $\mathrm{BCa}$ than others are needed. Another potential limitation was the residual confounding factors that had not been taken into account in the original studies. This concern was assessed with $E$-values. Importantly, the $E$-values of the effect estimates are small, indicating that our findings are not very robust and incontrovertible. In this respect, RCTs are invaluable for controlling various biases including residual confounding biases. Additionally, given that results from some sensitivity analyses did not support this evidence of the association, our findings should be interpreted with caution.

\section{Conclusion}

In conclusion, this meta-analysis adds to the body of evidence for an association between bisphosphonates and a decreased risk of primary $\mathrm{BCa}$. However, due to the lack of consistency between the results from observational studies and that from RCTs, future large-scale RCTs that focus on $\mathrm{BCa}$ primary prevention are needed.

\section{Key points}

Question: Does the use of bisphosphonates reduce the risk of primary breast cancer in women?

Findings: In this updated systematic review and metaanalysis that included five large cohorts, three large population-based case-control studies and two large-randomized controlled trials, in which 67,857 primary breast cancer patients and 188,685 bisphosphonate users involved, a $12 \%$ lower risk of primary breast cancer was associated with the use of bisphosphonates.

Meaning: These findings provide support for the use of bisphosphonates as $\mathrm{BCa}$ chemoprevention strategy, however, future randomized controlled trials with large sample sizes are required to validate this concern.

\section{Acknowledgments}

The authors acknowledge Justina Ucheojor Onwuka for her help in English language copy editing. This work was supported by The Seed Project Funds of Public Health School of Harbin Medical University (grant number 2012-05 to YL) and by a grant from The Harbin Medical University Innovation Research Foundation (2016JCZX14 to YL; 2016JCZX20 to YXZ). The funders and sponsors had no role in the design and conduct of the study; collection, management, analysis, and interpretation of the data; preparation, review, or approval of the manuscript; and decision to submit the manuscript for publication.

\section{Author contributions}

All authors had full access to the data in the study and take responsibility for the integrity of the data and accuracy of data analysis. YL, XZ and HS contributed equally to this work and should be considered co-first authors. Study concept and design: YL, SZ, YXZ, XZ, QZ and YSZ. Acquisition, analysis, or interpretation of data: YL, XZ, HS, SZ and YXZ. Drafting of the manuscript: YL, XZ, QZ and YSZ. Critical revision of the manuscript for important intellectual content: all authors. Statistical analysis: YL, SZ, HS, YXZ and YSZ. Administrative, technical, or material support: YL, QZ and YSZ. Study supervision: YL, QZ and YSZ. All authors contributed to data analysis, drafting or revising the article, gave final approval of the version to be published, and agree to be accountable for all aspects of the work. 


\section{Disclosure}

The authors report no conflicts of interest in this work.

\section{References}

1. Bray F, Ferlay J, Soerjomataram I, Siegel RL, Torre LA, Jemal A. Global cancer statistics 2018: GLOBOCAN estimates of incidence and mortality worldwide for 36 cancers in 185 countries. $C A$ Cancer J Clin. 2018. doi:10.3322/caac. 21492

2. Ferlay J, Soerjomataram I, Ervik M, et al. GLOBOCAN 2012 V1.1, Cancer Incidence and Mortality Worldwide: IARC CancerBase No. 11 [Internet]. Lyon, France: International Agency for Research on Cancer; 2014. Available from: http://globocan.iarc.fr, Accessed January 16, 2018

3. Rosen CJ. Clinical practice. Postmenopausal osteoporosis. $N$ Engl $J$ Med. 2005;353(6):595-603. doi:10.1056/NEJMcp043801

4. Watts NB, Lewiecki EM, Miller PD, Baim S. National osteoporosis foundation 2008 clinician's guide to prevention and treatment of osteoporosis and the world health organization Fracture Risk Assessment Tool (FRAX): what they mean to the bone densitometrist and bone technologist. J Clin Densitom. 2008;11(4):473-477. doi:10.1016/j.jocd.2008.04.003

5. Bedard PL, Body JJ, Piccart-Gebhart MJ. Sowing the soil for cure? Results of the ABCSG-12 trial open a new chapter in the evolving adjuvant bisphosphonate story in early breast cancer. J Clin Oncol. 2009;27(25):4043-4046. doi:10.1200/JCO.2008.21.4908

6. Green JR. Antitumor effects of bisphosphonates. Cancer. 2003;97(3 Suppl):840-847. doi:10.1002/cncr.11128

7. Wiemer AJ, Hohl RJ, Wiemer DF. The intermediate enzymes of isoprenoid metabolism as anticancer targets. Anticancer Agents Med Chem. 2009;9(5):526-542.

8. Santini D, Vincenzi B, Galluzzo S, et al. Repeated intermittent low-dose therapy with zoledronic acid induces an early, sustained, and long-lasting decrease of peripheral vascular endothelial growth factor levels in cancer patients. Clin Cancer Res. 2007;13(15 Pt 1):4482-4486. doi:10.1158/1078-0432.CCR-07-0551

9. Stresing V, Fournier PG, Bellahcene A, et al. Nitrogen-containing bisphosphonates can inhibit angiogenesis in vivo without the involvement of farnesyl pyrophosphate synthase. Bone. 2011;48(2):259-266. doi:10.1016/j.bone.2010.09.035

10. Dieli F, Gebbia N, Poccia F, et al. Induction of gammadelta T-lymphocyte effector functions by bisphosphonate zoledronic acid in cancer patients in vivo. Blood. 2003;102(6):2310-2311. doi:10.1182/blood-2003-05-1655

11. Benzaid I, Monkkonen H, Stresing V, et al. High phosphoantigen levels in bisphosphonate-treated human breast tumors promote Vgamma9Vdelta2 T-cell chemotaxis and cytotoxicity in vivo. Cancer Res. 2011;71(13):4562-4572. doi:10.1158/0008-5472.CAN-10-3862

12. Winter MC, Holen I, Coleman RE. Exploring the anti-tumour activity of bisphosphonates in early breast cancer. Cancer Treat Rev. 2008;34 (5):453-475. doi:10.1016/j.ctrv.2008.02.004

13. Clezardin P. Bisphosphonates' antitumor activity: an unravelled side of a multifaceted drug class. Bone. 2011;48(1):71-79. doi:10.1016/j. bone.2010.07.016

14. Hue TF, Cummings SR, Cauley JA, et al. Effect of bisphosphonate use on risk of postmenopausal breast cancer: results from the randomized clinical trials of alendronate and zoledronic Acid. JAMA Intern Med. 2014;174(10):1550-1557. doi:10.1001/jamainternmed.2014.3634

15. Liu Y, Zhao S, Chen W, et al. Bisphosphonate use and the risk of breast cancer: a meta-analysis of published literature. Clin Breast Cancer. 2012;12(4):276-281. doi:10.1016/j.clbc.2012.04.003

16. Chiang CH, Huang CC, Chan WL, et al. Oral alendronate use and risk of cancer in postmenopausal women with osteoporosis: anationwide study. J Bone Miner Res. 2012;27(9):1951-1958. doi:10.1002/jbmr.1645
17. Cardwell CR, Abnet CC, Veal P, Hughes CM, Cantwell MM, Murray LJ. Exposure to oral bisphosphonates and risk of cancer. Int J Cancer. 2012;131(5):E717-E725. doi:10.1002/ijc.27389

18. Fournier A, Mesrine S, Gelot A, et al. Use of bisphosphonates and risk of breast cancer in a French cohort of postmenopausal women. $J$ Clin Oncol. 2017;35(28):3230-3239. doi:10.1200/JCO.2016.71.4337

19. Vinogradova Y, Coupland C, Hippisley-Cox J. Exposure to bisphosphonates and risk of common non-gastrointestinal cancers: series of nested case-control studies using two primary-care databases. $\mathrm{Br}$ J Cancer. 2013;109(3):795-806. doi:10.1038/bjc.2013.383

20. Moher D, Liberati A, Tetzlaff J, Altman DG. Preferred reporting items for systematic reviews and meta-analyses: the PRISMA statement. Ann Intern Med. 2009;151(4):264-269.

21. Voskuil DW, Monninkhof EM, Elias SG, Vlems FA, van Leeuwen FE. Physical activity and endometrial cancer risk, a systematic review of current evidence. Cancer Epidemiol Biomarkers Prev. 2007;16 (4):639-648. doi:10.1158/1055-9965.EPI-06-0742

22. Jadad AR, Moore RA, Carroll D, et al. Assessing the quality of reports of randomized clinical trials: is blinding necessary? Control Clin Trials. 1996;17(1):1-12.

23. Higgins JP, Altman DG, Gotzsche PC, et al. The Cochrane Collaboration's tool for assessing risk of bias in randomised trials. Bmj. 2011;343:d5928. doi:10.1136/bmj.d5928

24. Vestergaard P, Fischer L, Mele M, Mosekilde L, Christiansen P. Use of bisphosphonates and risk of breast cancer. Calcif Tissue Int. 2011;88(4):255-262. doi:10.1007/s00223-011-9463-7

25. Chlebowski RT, Chen Z, Cauley JA, et al. Oral bisphosphonate use and breast cancer incidence in postmenopausal women. J Clin Oncol. 2010;28(22):3582-3590. doi:10.1200/JCO.2010.28.2095

26. Sun K, Liu JM, Sun HX, Lu N, Ning G. Bisphosphonate treatment and risk of esophageal cancer: a meta-analysis of observational studies. Osteoporos Int. 2013;24(1):279-286. doi:10.1007/s00198012-2158-8

27. Higgins JP, Thompson SG. Quantifying heterogeneity in a meta-analysis. Stat Med. 2002;21(11):1539-1558. doi:10.1002/ sim. 1186

28. Higgins JP, Thompson SG, Deeks JJ, Altman DG. Measuring inconsistency in meta-analyses. Bmj. 2003;327(7414):557-560. doi:10.1136/ bmj.327.7414.557

29. Greenland S. Quantitative methods in the review of epidemiologic literature. Epidemiol Rev. 1987;9:1-30. doi:10.1093/oxfordjournals. epirev.a036298

30. VanderWeele TJ, Ding P. Sensitivity analysis in observational research: introducing the E-value. Ann Intern Med. 2017;167 (4):268-274. doi:10.7326/M16-2607

31. Egger M, Davey Smith G, Schneider M, Minder C. Bias in meta-analysis detected by a simple, graphical test. Bmj. 1997;315 (7109):629-634. doi:10.1136/bmj.315.7109.629

32. Duval S, Tweedie R. Trim and fill: asimple funnel-plot-based method of testing and adjusting for publication bias in meta-analysis. Biometrics. 2000;56(2):455-463.

33. Newcomb PA, Trentham-Dietz A, Hampton JM. Bisphosphonates for osteoporosis treatment are associated with reduced breast cancer risk. Br J Cancer. 2010;102(5):799-802. doi:10.1038/sj. bjc. 6605555

34. Rennert G, Pinchev M, Rennert HS. Use of bisphosphonates and risk of postmenopausal breast cancer. J Clin Oncol. 2010;28 (22):3577-3581. doi:10.1200/JCO.2010.28.1113

35. Monsees GM, Malone KE, Tang MT, Newcomb PA, Li CI. Bisphosphonate use after estrogen receptor-positive breast cancer and risk of contralateral breast cancer. J Natl Cancer Inst. 2011;103 (23):1752-1760. doi:10.1093/jnci/djr399

36. Kwan ML, Shi JM, Habel LA, et al. Effectiveness of bisphosphonate use and risk of contralateral breast cancer and recurrence in women with early-stage breast cancer treated with tamoxifen. Breast Cancer Res Treat. 2016;156(2):379-389. doi:10.1007/s10549-016-3763-6 
37. Korde LA, Doody DR, Hsu L, Porter PL, Malone KE. Bisphosphonate use and risk of recurrence, second primary breast cancer, and breast cancer mortality in a population-based cohort of breast cancer patients. Cancer Epidemiol Biomarkers Prev. 2018;27 (2):165-173. doi:10.1158/1055-9965.EPI-17-0556

38. Wiseman M. The second world cancer research fund/american institute for cancer research expert report. Food, nutrition, physical activity, and the prevention of cancer: a global perspective. Proc Nutr Soc. 2008;67(3):253-256. doi:10.1017/S002966510800712X

39. Coleman RE, Rathbone E, Brown JE. Management of cancer treatment-induced bone loss. Nat Rev Rheumatol. 2013;9 (6):365-374. doi:10.1038/nrrheum.2013.36

40. Ou YJ, Chiu HF, Wong YH, Yang CC, Yang YH. Bisphosphonate use and the risk of breast cancer: a meta-analysis of observational studies. Pharmacoepidemiol Drug Saf. 2017;26(10):1286-1295. doi:10.1002/ pds.4302

41. Dreyfuss JH. Oral bisphosphonate use associated with a decreased risk of breast cancer. CA Cancer J Clin. 2010;60(6):343-344. doi:10.3322/caac. 20091

42. Black DM, Delmas PD, Eastell R, et al. Once-yearly zoledronic acid for treatment of postmenopausal osteoporosis. $N$ Engl J Med. 2007;356(18):1809-1822. doi:10.1056/NEJMoa067312

43. Early Breast Cancer Trialists' Collaborative G. Adjuvant bisphosphonate treatment in early breast cancer: meta-analyses of individual patient data from randomised trials. Lancet. 2015;386 (10001):1353-1361. doi:10.1016/S0140-6736(15)60908-4

44. Russell RG, Watts NB, Ebetino FH, Rogers MJ. Mechanisms of action of bisphosphonates: similarities and differences and their potential influence on clinical efficacy. Osteoporos Int. 2008;19(6):733-759. doi:10.1007/ s00198-007-0540-8

45. Licata AA. Discovery, clinical development, and therapeutic uses of bisphosphonates. Ann Pharmacother. 2005;39(4):668-677. doi:10.1345/ aph.1E357

46. Hollick RJ, Reid DM. Role of bisphosphonates in the management of postmenopausal osteoporosis: an update on recent safety anxieties. Menopause Int. 2011;17(2):66-72. doi:10.1258/mi.2011.011014
47. Reid IR, Cornish J. Epidemiology and pathogenesis of osteonecrosis of the jaw. Nat Rev Rheumatol. 2012;8(2):90-96. doi:10.1038/ nrrheum.2011.181

48. Andrici J, Tio M, Eslick GD. Meta-analysis: oral bisphosphonates and the risk of oesophageal cancer. Aliment Pharmacol Ther. 2012;36 (8):708-716. doi:10.1111/apt.12041

49. Oh YH, Yoon C, Park SM. Bisphosphonate use and gastrointestinal tract cancer risk: meta-analysis of observational studies. World J Gastroenterol. 2012;18(40):5779-5788. doi:10.3748/wjg.v18.i40.5779

50. Hermann AP, Abrahamsen B. The bisphosphonates: risks and benefits of long term use. Curr Opin Pharmacol. 2013;13(3):435-439. doi:10.1016/j.coph.2013.02.002

51. Dreyfuss JH. Tamoxifen infrequently used by women at risk for breast cancer. CA Cancer J Clin. 2010;60(4):204-206. doi:10.3322/caac.20080

52. Powles TJ. Extended adjuvant tamoxifen for breast cancer - anew era? Lancet. 2013;381(9869):782-783. doi:10.1016/S0140-6736(12) 62038-8

53. Davies C, Pan H, Godwin J, et al. Long-term effects of continuing adjuvant tamoxifen to 10 years versus stopping at 5 years after diagnosis of oestrogen receptor-positive breast cancer: ATLAS, a randomised trial. Lancet. 2013;381(9869):805-816. doi:10.1016/ S0140-6736(12)61963-1

54. Waters EA, Cronin KA, Graubard BI, Han PK, Freedman AN. Prevalence of tamoxifen use for breast cancer chemoprevention among U.S. women. Cancer Epidemiol Biomarkers Prev. 2010;19 (2):443-446. doi:10.1158/1055-9965.EPI-09-0930

55. Kirk R. Oral bisphosphonate use may protect women from breast cancer. Nat Rev Clin Oncol. 2010;7(9):482. doi:10.1038/nrclinonc.2010.126

56. Coleman R, Gnant M, Morgan G, Clezardin P. Effects of bone-targeted agents on cancer progression and mortality. J Natl Cancer Inst. 2012;104(14):1059-1067. doi:10.1093/jnci/djs263

57. Gnant M, Clezardin P. Direct and indirect anticancer activity of bisphosphonates: a brief review of published literature. Cancer Treat Rev. 2012;38(5):407-415. doi:10.1016/j.ctrv.2011.09.003
Clinical Epidemiology

\section{Publish your work in this journal}

Clinical Epidemiology is an international, peer-reviewed, open access, online journal focusing on disease and drug epidemiology, identification of risk factors and screening procedures to develop optimal preventative initiatives and programs. Specific topics include: diagnosis, prognosis, treatment, screening, prevention, risk factor modification, systematic reviews, risk \& safety of medical interventions, epidemiology \& biostatistical methods, and evaluation of guidelines, translational medicine, health policies \& economic evaluations. The manuscript management system is completely online and includes a very quick and fair peer-review system, which is all easy to use. 